\section{Enfermedad de Dupuytren: revisión}

\author{
PABLO WAGNER ${ }^{\text {a }}$, JAVIER A. ROMÁN, JORGE VERGARA
}

\section{Dupuytren disease}

Dupuytren disease (DD) is a connective tissue disorder that consists in fibromatosis of the palmar and digital fascia (in form of nodules or flanges) that leads to the development of flexion contractures of the palm and fingers. The little and ring finger are particularly affected. The disease can limit hand function, reducing the quality of life. The disease can have a traumatic origin and is also associated with conditions such as diabetes mellitus, alcoholism, dyslipidemia, epilepsy and AIDS, among others. However, none of these conditions can fully explain the genesis of DD. A hereditary component is described in $40 \%$ of patients and is attributed to an autosomal dominant gene of variable penetrance, probably related to collagen synthesis. However there are also spontaneous and recessive inheritance cases. The diagnosis is clinical and based on physical examination. Treatment ranges from observation or use of injectable collagenase to the surgical option in cases with significant functional limitations.

(Rev Med Chile 2012; 140: 1185-1190).

Key words: Collagenasses; Dupuytren contracture; Heredity.

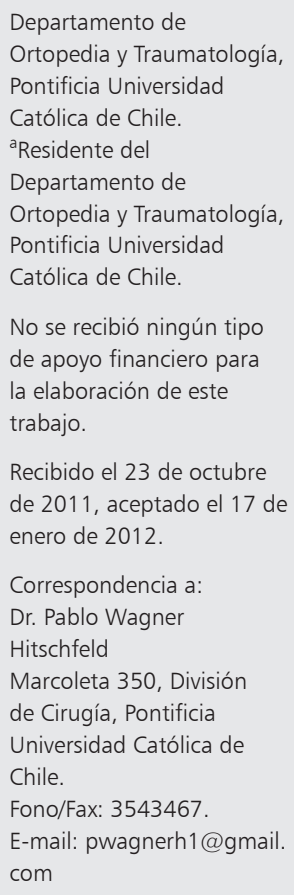

L a enfermedad de Dupuytren (ED) es una condición benigna del tejido conectivo, caracterizado por un desorden fibroproliferativo (fibromatosis benigna) que afecta fundamentalmente a la aponeurosis palmar y digital, provocando contracturas y deformidad progresiva en flexión en la región palmar de mano y dedos, cuya causa y mecanismo fisiopatológico aún permanecen parcialmente comprendidos.

El primer caso de esta condición fue descrito por el médico suizo Félix Plater en 1614 (quien le atribuyó una causa traumática), siendo luego descrito por otros autores como H Cline, en 1777 y por Ashley Cooper, en 1822; sin embargo, el nombre del médico francés Guillaume Dupuytren predominó para esta condición, quien en 1831 describe en detalle la fibromatosis localizada en la aponeurosis palmar, realizando la primera cirugía de fasciotomía abierta.

Esta enfermedad se caracteriza por el desarrollo de contracturas en flexión, de la palma y de las articulaciones digitales, secundaria a la formación de nódulos y bandas fibrosas o bridas ${ }^{1-5}$.

Afecta principalmente la región palmar distal en relación a los dedos anular y meñique, produ- ciendo la flexión progresiva e irreversible de las articulaciones metacarpo-falángicas (MTCF) e interfalángicas proximales (IFP).

\section{Epidemiología}

En relación a la prevalencia de esta enfermedad, destaca que es un cuadro que sucede en toda la población, pero en diferente proporción y frecuencia dependiendo de la carga genética y de las comorbilidades del paciente. La incidencia de esta patología aparece mayor en los países del norte de Europa y emigrantes provenientes de esas zonas; mientras que es rara en países africanos y Asia oriental. La prevalencia reportada en otros países es de 1,7 a $2 \%$ en varones adultos mayores de 50 años en la población general, siendo de 3-6\% en adultos caucásicos. En Escocia, Noruega e Islandia se ha reportado una incidencia de hasta $40 \%$ en ciertas comunidades, mientras que su presencia en poblaciones de raza negra y de Asia oriental es de escasa ocurrencia. Es siete a diez veces más frecuente en hombres que en mujeres. Para esto aún no hay explicación, sin embargo los miofibroblastos en la ED tienen una mayor expresión de 
receptores para andrógenos que los fibroblastos en sujetos sanos, lo que podría explicar en parte la predominancia en el género masculino ${ }^{3}$. Cuando se presenta en mujeres la $\mathrm{ED}$ es de más rápida progresión que en hombres, hecho no aclarado en la literatura. Se presenta en forma bilateral hasta en $70 \%$ de los casos siendo de predominancia en la mano derecha $(60 \%)^{4,5}$. En los hombres la edad de presentación media es habitualmente una década antes que la edad presentación media en mujeres.

\section{Patogenia}

Sobre su patogénesis se describen 3 fases. La fase proliferativa, involutiva y la tardía o residual. La fase proliferativa se caracteriza por el desarrollo de nódulos compuestos por fibroblastos y colágeno tipo III. La fase involutiva se caracteriza por una conducta contráctil del tejido debido al reemplazo de los fibroblastos por miofibroblastos, desarrollándose cordones fácilmente palpables bajo la piel. Por último, en la fase tardía o residual, los nódulos regresan persistiendo sólo el componente contráctil evolucionando a una flexión progresiva de la articulación MTCF y la IFP ${ }^{4-6}$. Estas fases no tienen gran importancia en el tratamiento, ya que principalmente lo que define es la ocurrencia o no de contracción de la MTCF.

\section{Etiología}

La etiología de la contractura de Dupuytren es desconocida. El origen traumático inicialmente postulado como causante ha ido cayendo en descrédito, debido a la ausencia de asociación con la mano dominante, con algún tipo de trabajo específico y la fuerte asociación familiar y genética descrita. Se consigue descubrir un componente hereditario en hasta $40 \%$ de los pacientes. Algunos lo han atribuido a un gen de herencia autosómico dominante, de penetrancia variable, donde el gen alterado se relaciona probablemente con la síntesis de colágeno.

Goyrand fue el primero que describió la asociación familiar ya en 1833. Además la fuerte variabilidad en prevalencia según geografía es una fuerte evidencia de una susceptibilidad heredable. En muchos pedigrees la heredabilidad aparenta seguir un modelo autosómico dominante, descrito por Skoog y Stackebrandt ya en los años 30 y 40. Uno de los escasos estudios con mayor población se realizó en Edimburgo, por Ling, que mostró que la enfermedad estaba presente en los mayores de 60 años en 53\% de los hombres y en 33\% de las mujeres ${ }^{2,7}$.

Estudios genéticos se han realizado en pacientes con $\mathrm{ED}$, no encontrándose aún mutaciones genéticas que se relacionen directamente con la $\mathrm{ED}$, pero sí se han descrito SNPs (single nucleotide polymorphismos o polimorfismos de nucleótido simple) que son alteraciones genéticas mínimas que aportan una mayor susceptibilidad a tener la ED. La mayoría de estos polimorfismos se localizan en genes de los cromosomas 6,11 y 16 que codifican a proteínas en la vía de señalización $\mathrm{Wnt}^{2,8-10}$.

Otros estudios han implicado el TGF-b1 como gen de susceptibilidad ${ }^{10-12}$. Sin embargo, estudios de la asociación entre los polimorfismos de nucleótidos de este gen con la incidencia de ED no han podido aclarar el asunto.

Lo descrito como una herencia autosómica dominante, es referido por algunos autores sólo como una hipótesis informada. Una herencia autosómica recesiva es también posible, pudiendo ser concordante con algunos casos familiares. También hay muchos casos esporádicos, sin aparente herencia caucásica europea, que mantienen en duda si estos casos tienen una base genética.

Como se puede ver de lo recién expuesto, la alteración genética y el mecanismo específico aún no se ha encontrado, pudiendo tratarse de una enfermedad de origen monogénica, poligénica o de componente variable entre una interacción genética y ambiental.

Respecto a las asociaciones con otros factores se describen múltiples elementos y algo ya se mencionó sobre el trauma o trabajo manual. Este último no se ha logrado relacionar de forma clara con la génesis ni sintomatología de la $\mathrm{ED}^{13,14}$. No hay una diferencia significativa en la lateralidad de la afección de la mano dominante versus la no dominante. Sí habría una relación de la exposición a vibración con la ED, incluso en términos de dosisrespuesta ${ }^{13,15}$. Sobre una lesión traumática como causa de la ED hay hallazgos contradictorios en la literatura. Se menciona que en sujetos predispuestos genéticamente un trauma podría contribuir a la aparición de la enfermedad. Sin embargo, para aceptar esta hipótesis como plausible la lesión debería aparecer en el lugar afectado no más allá de 2 años después de la lesión y con evidencia clara de que hubo tal traumatismo. No se ha podido 
aclarar la relación entre daño hepático crónico por alcohol ni consumo de alcohol con la ED. Otro elemento considerado como posible asociación es el tabaquismo, el que se ha encontrado que tiene una relación estadísticamente significativa con la $\mathrm{ED}$, sugiriéndose que podría estar relacionado en la patogenia de la enfermedad por una oclusión microvascular con fibrosis y posterior contracción de las fascia palmar/digital ${ }^{16-18}$.

Respecto a la relación con otras enfermedades, clásicamente se ha descrito la relación con la diabetes mellitus (DM). Estudios que evaluaron la relación con la DM tipo 1, encontraron relación entre la de edad de inicio y la duración de la DM con la génesis de la enfermedad de Dupuytren ${ }^{19,20}$. En DM tipo 2 se encontró relación con la aparición de macroalbuminuria y con las glicemias de ayuno alteradas, lo que se correlaciona con un DM mal controlada (pero sin detallar los mecanismos e influencia de glicemias elevadas en estos tejidos específicos de la mano). En la artritis reumatoidea se ha encontrado su asociación negativa con la enfermedad ${ }^{21,22}$, estando aun el fundamento en estudio. La enfermedad de Peyronie (fibromatosis de los cuerpos cavernosos del pene) también se ha relacionado con la $\mathrm{ED}$, encontrándose afección de las manos en $20 \%$ de los pacientes con Peyronie. También existe la observación inversa, al presentarse la enfermedad de Peyronie en 3\% de los pacientes con $\mathrm{ED}^{23}$. Respecto a la dislipidemia (DLP), hay hallazgos que sugieren una influencia de ésta en la etiopatogenia de la ED, sin embargo debe tenerse presente la alta frecuencia de DLP en pacientes diabéticos, alcohólicos, entre otros.

\section{Diagnóstico}

En general es un cuadro de diagnóstico tardío ya que inicialmente la enfermedad se va a manifestar sólo como pequeñas depresiones de la palma, o bien como zonas con cambio de la pigmentación o fibrosas. Posteriormente aparecen los nódulos, que son de tejido blando, en general ubicado a nivel del pliegue palmar distal, pero también puede aparecer en los dedos, a nivel de la articulación interfalángica proximal (más común en los dedos meñique y anular). En general estos nódulos son asintomáticos, asociándose en ocasiones a tenosinovitis estenosante (si es que hay compromiso de la polea de los flexores, impidiendo el deslizamiento de los mismos bajo la polea). Sobre la cara dorsal de las articulaciones interfalángicas pueden aparecer nódulos indurados, llamados nódulos de Garrod, que se asocian a enfermedad de Peyronie ${ }^{23}$, Ledderhose (fibromatosis plantar) y a una ED de mayor gravedad y avance (diátesis de Dupuytren). Posteriormente aparecen bridas o cordones, que producen una retracción progresiva de los dedos comprometidos hacia la postura en flexión de los mismos. Puede aparecer disminución del espacio interdigital, alteraciones de eje axial como rotacional y disfunción tanto estática como dinámica del dedo comprometido.

Una prueba a realizar al examen físico es la prueba de Hueston (test de apoyo de mano en la mesa), que se realiza apoyando la palma sobre una superficie plana, debiendo ser capaz de apoyar todos los dedos extendidos de una forma uniforme. Este test es positivo cuando no se consigue apoyar todos los dedos extendidos sobre la mesa y evidencia una contractura en flexión de alguno de los dedos. La presencia de un test positivo es predictor del beneficio de la cirugía, ya que la positividad de este test representa la angulación de por lo menos $30^{\circ}$ de la articulación metacarpofalángica, situación en la cual los pacientes operados responden satisfactoriamente a la pregunta si la cirugía les modificó la función de la mano.

Dentro del diagnóstico diferencial se deben mencionar las contracturas dérmicas por quemaduras, contracturas aisladas de los dígitos por lesiones del aparato flexo-extensor y un cuadro llamado contractura no Dupuytren. Este último fue descrito por Anderson en $1891^{24}$, y consiste en la contractura de las partes blandas profundas de la mano, que se da principalmente en razas aborígenes más que en razas blancas, no tiene historia familiar, no hay predilección por género, el compromiso de la palma es en línea con un solo dedo, pero sin comprometerlo, no hay contractura de las articulaciones digitales y la mayoría de las veces sólo aparece en respuesta a traumas o intervenciones quirúrgicas de la palma. Tiene una resolución espontánea en la mayoría de los casos, es de curso benigno y en contadas ocasiones requiere cirugía ${ }^{25}$.

En relación al estudio diagnóstico, no se recomienda ningún examen de imagen. Sin embargo, en caso de duda diagnóstica es planteable la ecografía de partes blandas, sin embargo, en nuestro centro no es de uso rutinario.

Respecto a clasificaciones, la más usada actual- 
mente en clínica divide la enfermedad en 4 etapas, siendo la etapa I la retracción secundaria de la articulación MTC-F del dedo anular, la etapa II se agrega la retracción de la IFP del anular más la contracción MTC-F del meñique, en la etapa III se agregan las interfalángicas del anular y meñique con la MTC-F del dedo medio y en la etapa IV se agrega hiperextensión de la IFD del meñique.

\section{Tratamiento}

\section{Tratamiento no quirúrgico}

La observación es planteable en casos leves, no progresivos, no limitantes de la funcionalidad. Se ha reportado el uso de la radioterapia, principalmente en uso en países europeos, con buena respuesta, retardando y enlenteciendo la progresión de la enfermedad, retrasando por lo tanto la necesidad de tratamiento quirúrgico ${ }^{26}$. Históricamente podemos mencionar que se han utilizado inyecciones de corticoesteroides, dimetilsulfóxido, vitamina A y E e interferón gamma, sin resultados consistentes ${ }^{27-30}$.

Sí se han obtenido resultados favorables con inyecciones de colagenasas. Los primeros trabajos de tratamiento con sustancias degradadoras del colágeno aparecen con Hueston en 1971, quien acuño el término de fasciotomía enzimática y utilizó para ello una mezcla de tripsina, hialuronidasa y lidocaína, sin embargo su trabajo no tuvo grandes seguidores. La purificación de la colagenasa específica derivada del Clostridium histolyticum se debe a Maclennon, en 1953, quien publica un artículo sobre la destrucción bacteriana del colágeno; sin embargo su uso en el tratamiento de la ED se remonta tan sólo a unos 15 años atrás, con los primeros estudios in vitro. Se reconocen más de 7 tipos de colagenasas del clostridium histolyticum, las cuales se pueden agrupar en 2 clases. Estas metaloproteasas se unen y quiebran la estructura tridimensional del colágeno. La FDA ha aprobado en febrero de 2010 el compuesto llamado Xiaflex ${ }^{\oplus}$ Xiapex $^{\oplus}$ en Europa) para el tratamiento de la ED. El medicamento se inyecta directamente en la cuerda pretendinea, pudiendo ser utilizado para la contractura en flexión de la articulación metacarpofalángica como para la articulación interfalángica proximal.

Se han realizado estudios prospectivos, comparados con placebo, con seguimiento hasta 2 años $^{30-32}$ así como estudios retrospectivos con mayores seguimientos $(8 \text { años })^{33}$, que muestran una efectividad de aproximadamente $65 \%$ en la resolución completa $\left(0-5^{\circ}\right.$ de contractura remanente) de la contractura con el uso de esta enzima con un máximo de 3 inyecciones. Se puede utilizar en las contracturas de cualquier grado, sin embargo, hay una mejor respuesta en las articulaciones MTCF que en las IFP y mientras mayor sea la contractura inicial de la articulación, peor es la respuesta a la inyección. El porcentaje de recurrencia no se conoce con exactitud, sin embargo este se reporta a partir del primer año de seguimiento en un $10 \%$, siendo progresivo hasta en $60 \%$ a los 8 años de seguimiento (datos retrospectivos $)^{33}$. Respecto a la gravedad de la recurrencia esta es siempre de menor magnitud que la contractura inicial. Efectos adversos graves (rotura tendínea, rotura de poleas, compromiso neurológico, reacción alérgica sistémica) ocurren en aproximadamente $1 \%$ de los pacientes. Efectos adversos transitorios como edema, prurito, dolor en sitio de punción, adenopatía axilar palpable dolorosa, inflamación local, entre otros, ocurren en $97 \%$ de los pacientes cediendo completamente a la semana post punción. Este tratamiento aún no está disponible en Chile.

\section{Tratamiento quirúrgico}

La cirugía es el estándar de oro para el tratamiento de las formas progresivas de la enfermedad. Este se plantea en casos de una contracción de la articulación metacarpo falángica (MTCF) de $30^{\circ}$ o más (lo que se considera una prueba de Hueston positiva) o contracción de la interfalángica proximal asociada a un deterioro funcional, habitualmente presente con contracturas mayores a $20^{\circ}$. La cirugía consiste principalmente en alguna de las siguientes opciones: fasciotomía percutánea, fasciectomía abierta parcial, fasciectomía abierta total, dermofasciectomía y amputación.

La fasciotomía percutánea es la técnica menos invasiva de todas, se emplea en caso de pacientes que no son buenos candidatos para una intervención quirúrgica mayor, especialmente adultos mayores con múltiples comorbilidades, ya que puede conseguirse resultados inmediatos en la extensión de los dedos con un mínimo riesgo al usar sólo anestesia local. Se realiza habitualmente con una aguja con la que se secciona la cuerda fibrosa en forma percutánea. Un estudio reciente comparando esta técnica con fasciectomía abierta 
a las 6 semanas de evolución demostró una mejoría del déficit de extensión pasivo de $63 \%$, comparado con una mejoría de 79\% del grupo de fasciectomía abierta. Respecto a las complicaciones hubo un mayor número de complicaciones menores (lesiones de piel y parestesias) en el grupo percutáneo versus fasciectomía, sin embargo el grupo percutáneo no tuvo complicaciones mayores, las que si se presentaron en el grupo quirúrgico (lesión de nervio digital, infección y hematoma $)^{34,35}$.

La fasciectomía parcial es el método quirúrgico más utilizado y aceptado en todo el mundo, y consiste en la escisión quirúrgica sólo del tejido macroscópicamente comprometido, lográndose una baja tasa de complicaciones y morbilidad post-operatoria. Sin embargo, los valores sobre su tasa de recurrencia pueden ir desde 27 a $63 \%{ }^{36-39}$.

La fasciectomía total o radical que consiste en la escisión completa de toda la fascia palmar, siendo un procedimiento con una alta tasa de complicaciones post-operatorias, con $20-30 \%$ de complicaciones mayores como hematomas, retrasos de cicatrización y rigidez articular entre otros. No se han demostrado mejores resultados clínicos ni de recidiva cuando se los ha comparado con la fasciectomía parcial. Sin embargo, si hay estudios que la avalan ${ }^{40}$, donde publican $18 \%$ de complicaciones (de los cuales $4 \%$ son lesiones nerviosas) y $11 \%$ de recurrencias. Claramente influye la experiencia de cada centro en los resultados.

La dermofasciectomía es un procedimiento de uso en pacientes con alto riesgo de recidiva y progresión, en el cual se realiza una extirpación de la piel y tejidos subyacentes con un injerto posterior. Tiene una baja probabilidad de recidiva, pero si mayores complicaciones como anestesia en la zona del injerto de piel total, hematomas, infección, entre otros ${ }^{36}$.

Finalmente la amputación es una indicación de excepción, en casos de múltiples recurrencias, con dedos sin función, no corregibles por la cirugía y sin un requerimiento funcional importante. Esto es más frecuente cuando existe una grave contractura en flexión del meñique.

\section{Pauta de manejo y derivación}

Una vez que se ha realizado el diagnóstico, se debe evaluar la velocidad de progresión, el grado de contractura articular y de déficit funcional que tiene el paciente. En caso de ser una enfermedad de lenta progresión (sin cambios en últimos 5 años), con prueba de Hueston (-) y que no produce un déficit funcional importante para el paciente, es planteable la observación clínica con control anual. En caso de ser un cuadro de más rápida progresión, test de Hueston (+) y que produce un déficit funcional al paciente, se debe derivar al especialista. Este decidirá en conjunto con el paciente el tratamiento a seguir, que probablemente será quirúrgico (fasciectomía parcial). Una vez que las colagenasas estén disponibles en Chile, estas serán una excelente terapia alternativa a la cirugía en casos primarios (no recidiva) y de menor gravedad.

\section{Referencias}

1. Gudmundsson KG, Jónsson T, Arngrímsson R; Guillaume Dupuytren and finger contractures. The Lancet, 2003; 362: 165-8.

2. Burge P. Genetics of Dupuytren's disease. Hand Clin 1999; 15: 63-71.

3. Hughes TB Jr, Mechrefe A, Littler JW, Akelman E. Dupuytren's disease. Journal of the American Society for Surgery of the Hand 2003; 3: 27-40

4. Ghazi M. Rayan, Dupuytren Disease: Anatomy, Pathology, Presentation, and Treatment. J Bone Joint Surg Am 2007; 89: 189-98.

5. Thurston AJ, Dupuytren's disease, J Bone Joint Surg [Br] 2003; 85-B: 469-77.

6. Rayan GM, Parizi M, Tomasek JJ. Pharmacologic regulation of Dupuytren's fibroblast contraction in vitro. J Hand Surg Am 1996; 21 (6): 1065-70.

7. Ling RSM. The genetic factor in Dupuytren's disease. J Bone Joint Surg [Br] 1963; 45-B: 709-18.

8. Ojwang JO, Adrianto I, Gray-McGuire C, Nath SK, Harley JB, Rayan GM. Genome-Wide Association Scan of Dupuytren's Disease. Hand Surg Am 2010; 35 (12): 2039-45.

9. Dolmans GH, Werker PM, Hennies HC, Furniss D, Festen EA, Franke L. Wnt Signaling and Dupuytren's Disease. N Engl J Med 2011; 365: 307-17.

10. Mosakhani N, Guled M, Lahti L, Borze I, Forsman M, Pa"a"kko"nen V, et al. Unique microRNA profile in Dupuytren's contracture supports deregulation of bcatenin pathway. Modern Pathology 2010; 23: 1544-52.

11. Bayat A, Alansar A, Hajeer AH, et al. Genetic susceptibility in Dupuyren's disease: lack of association of a novel transforming growth factor beta(2) polymorphism in Dupuytren's disease. J Hand Surg [Br] 2002; 27-B: 47-9. 
12. Al-Qattan MM. Factors in the Pathogenesis of Dupuytren's Contracture. J Hand Surg 2006; 31A: 1527 34.

13. Liss GM, Stock SR. Can Dupuytren's contracture be work-related? Review of the evidence. Am J Ind Med 1996; 29: 521-32.

14. Thomas PR, Clarke D. Vibration white finger and Dupuytren's contracture: are they related? Occup Med (Lond) 1992; 42: 155-8.

15. Bovenzi M. Hand-arm vibration syndrome and doseresponse relation for vibration induced white finger among quarry drillers and stonecarvers. Italian Study Group on Physical Hazards in the Stone Industry. Occup Environ Med 1994; 51: 603-11.

16. Fraser-Moodie A. Dupuytren's contracture and cigarette smoking. Br J Plastic Surg 1976; 29: 214-5.

17. An H, Southworth S, Jackson W, Russ B. Cigarette smoking and Dupuytren's contracture of the hand. J Hand Surg [Am] 1988; 13- A: 872-3.

18. Eadington DW, Patrock AW, Frier BM. Association between connective tissue changes and smoking habit in type 2 diabetes and in nondiabetic humans. Diabetes Res Clin Pract Suppl 1991; 11: 121-598-9.

19. Arkkila PE, Kantola IM, Viikari JS. Dupuytren's disease: Association with Chronic diabetic complication. J Rheumatol 1997; 24 (1): 153-9.

20. Arkkila PE, Kantola I, Viikari J, Ronnemaa T, Vahatalo MA. Dupuytren's disease in type 1 diabetic patients: a five-year prospective study. Clin Exp Rheumatol 1996; 14: 59-65.

21. Arafa M, Steingold RF, Noble J. The incidence of Dupuytren's disease in patients with rheumatoid arthritis. J Hand Surg [Br] 1984; 9- B: 165-6.

22. Gudmundsson K, Arngrimsson R, Sigfusson N, et al. Prevalence of joint complaints amongst individuals with Dupuytren's disease: from the Reykjavik study. Scand J Rheumatol 1999; 28: 300-4.

23. Hindocha S, Stanley JK, Watson S, Bayat A. Dupuytren's Diathesis Revisited: Evaluation of Prognostic Indicators for Risk of Disease Recurrence J Hand Surg 2006; 31A: 1626-34.

24. Anderson W. Lectures on contractures of the fingers and toes: their varieties, pathology and treatment. Lancet 1891; 2: 57-9.

25. Rayan GM, Moore J. Non-Dupuytren's disease of the palmar fascia. J Hand Surg Br 2005; 30 (6): 551-6.

26. Seegenschmiedt M, H-B Makoski H-B,Trott K-R, Brady L.(Eds.) Radiotherapy for Non-Malignant Disorders Springer (Berlin, New York, 2008) chapter 9, "Morbus
Dupuytren / Morbus Ledderhose" (p 161-92).

27. Meek RM, McLellan S, Crossan JF. Dupuytren's disease. A model for the mechanism of fibrosis and its modulation by steroids. J Bone Joint Surg Br 1999; 81: 732-8.

28. Meek RM, McLellan S, Reilly J, Crossan JF.The effect of steroids on Dupuytren's disease: role of programmed cell death J Hand Surg Br 2002; 27 (3): 270-3.

29. Pittet B, Rubbia-Brandt L, Desmoulière A, Sappino AP, Roggero P, Guerret S, et al. Effect of gamma-interferon on the clinical and biologic evolution of hypertrophic scars and Dupuytren's disease: an open pilot study. Plast Reconstr Surg 1994; 94 (7): 1100-1.

30. Badalamente MA, Hurst LC. Efficacy and Safety of Injectable Mixed Collagenase Subtypes in the Treatment of Dupuytren's Contracture. The Journal of Hand Surgery 2007; 32A (6): 767-74.

31. Hurst LC, Badalamente MA, Hentz VR, Hotchkiss RN, Kaplan TD, Meals RA, et al. Inyectable conllagenase clostridium histolyticum for dupuytren's contracture. N Engl J Med 2009; 361: 968-79 (CORD I).

32. Gilpin D, Coleman S, Hall S, Houston A, Karrasch J, Jones N. Inyectable collagenase Clostridium Histolyticum: A new nonsurgical treatment for Dupuytren's disease. J Hand Surg 2010; 35A: 2027-38. (CORD II).

33. Watt AJ, Curtin CM, Hentz VR. Collagenase injection as nonsurgical treatment of Dupuytren's disease: 8-year follow up. J Hand Surg 2010; 35A: 534-9.

34. Desai SS, Hentz VR. Treatment of Dupuytren disease. J Hand Surg 2011; 36A: 936-42.

35. Van Rijssen AL, Gerbrandy FS, Linden HT, Klip H, Werker PMN. A Comparison of the Direct Outcomes of Percutaneous Needle Fasciotomy and Limited Fasciectomy for Dupuytren's Disease: A 6-Week Follow-Up Study. J Hand Surg 2006; 31A: 717-25.

36. Stanbury SJ, Hammert WC. Dupuytren Contracture. Article in press. JHS, 2011.

37. Makela EA, Jaroma H, Harju A, Anttila S, Vainio J. Dupuytren's contracture: the long term results after day surgery. J Hand Surg [Br] 1991; 16: 272-4.

38. Rodrigo JJ, Niebauer JJ, Brown RL, Doyle JR. Treatment of Dupuytren's contracture. Long term results after fasciotomy and fascial excision. J Bone Joint Surg [Am] 1976; 58: 380-7.

39. Brandt KE. An Evidence-Based Approach to Dupuytren's Contracture. Plast Reconstr Surg 2010; 126 (6): 2210-5.

40. Högemann A, Wolfhard U, Kendoff D, Board TN, Olivier LC. Results of total aponeurectomy for Dupuytren's contracture in 61 patients: a retrospective clinical study. Arch ORthop Trauma Surg (2009) 129: 195-201. 